\title{
Erratum to: The Presence in tRNA Molecule Sequences of the Double Hairpin, an Evolutionary Stage Through Which the Origin of this Molecule is Thought to have Passed
}

\author{
Sergio Branciamore • Massimo Di Giulio
}

Published online: 27 April 2011

(C) Springer Science+Business Media, LLC 2011

Erratum to: J Mol Evol

DOI 10.1007/s00239-011-9440-9

The paper was published erroneously with error in Appendix tables, values of CL were erroneously inverted. Corrected tables are given overleaf.

The online version of the original article can be found under doi:10.1007/s00239-011-9440-9.

S. Branciamore $\cdot$ M. Di Giulio

Department of Molecular and Cellular Biology, Beckman

Research Institute, City of Hope, Duarte, CA 91010, USA

M. Di Giulio ( $\square)$

Laboratory for Molecular Evolution, Institute of Genetics

and Biophysics 'Adriano Buzzati Traverso', CNR,

Via P. Castellino, 111, 80131 Naples, Napoli, Italy

e-mail: digiulio@igb.cnr.it 
Table 9 Top 50 stable DH structures in actual tRNA sequences

\begin{tabular}{|c|c|c|c|}
\hline geneID & $\begin{array}{l}\mathrm{CL} \Delta G \\
(\mathrm{kcal} / \mathrm{mol})\end{array}$ & $\begin{array}{l}\mathrm{DH} \Delta G \\
(\mathrm{kcal} / \mathrm{mol})\end{array}$ & $\begin{array}{l}\text { Expected CI/CL } \\
\text { frequency ratio }\end{array}$ \\
\hline >tdbD00012005|Nanoarchaeum_equitans_Kin4-M|228908|Pro|GGG & -39.8 & -31 & $1.32 \times 10^{-7}$ \\
\hline >tdbD00003796|Aeropyrum_pernix_K1|272557|Ala|TGC & -34.9 & -29.6 & $3.17 \times 10^{-4}$ \\
\hline >tdbD00005218|Aquifex_aeolicus_VF5|224324|Gly|TCC & -36.9 & -29.4 & $4.59 \times 10^{-5}$ \\
\hline >tdbD00010376|Thermotoga_maritima_MSB8|243274|Thr|GGT & -33.3 & -29.4 & $6.59 \times 10^{-7}$ \\
\hline >tdbD00008351|Coxiella_burnetii_RSA_493|227377|Pro|CGG & -31.5 & -27.8 & $1.49 \times 10^{-7}$ \\
\hline >tdbD00005163|Mycobacterium_leprae_TN|272631|Gly|GCC & -32 & -27.8 & $5.84 \times 10^{-6}$ \\
\hline >tdbD00005150|Mycobacterium_tuberculosis_CDC1551|83331|Gly|GCC & -32 & -27.8 & $2.02 \times 10^{-5}$ \\
\hline >tdbD00005468|Mycobacterium_bovis_AF2122/97|233413|Gly|GCC & -32 & -27.8 & $6.47 \times 10^{-6}$ \\
\hline >tdbD00005519|Mycobacterium_tuberculosis_H37Rv|83332|Gly|GCC & -32 & -27.8 & $4.14 \times 10^{-3}$ \\
\hline >tdbD00000227|Caenorhabditis_elegans|6239|Ala|AGC & -31.4 & -27.7 & $4.25 \times 10^{-11}$ \\
\hline >tdbD00010503|Caenorhabditis_elegans|6239|Thr|AGT & -31.4 & -27.7 & $3.16 \times 10^{-9}$ \\
\hline >tdbD00003877|Caenorhabditis_elegans|6239|AlalAGC & -31.4 & -27.7 & $3.16 \times 10^{-9}$ \\
\hline >tdbD00003875|Caenorhabditis_elegans|6239|AlalAGC & -31.4 & -27.7 & $9.06 \times 10^{-7}$ \\
\hline$>$ tdbD00005416|Leptospira_interrogans_serovar_Lai_str._56601|189518|Gly|GCC & -33 & -27.6 & $4.87 \times 10^{-5}$ \\
\hline >tdbD00012049|Staphylothermus_marinus_F1|399550|Met|CAT & -38.8 & -27.6 & $4.87 \times 10^{-5}$ \\
\hline >tdbD00011436|Rhodopseudomonas_palustris_CGA009|258594|Trp|CCA & -28.8 & -27.5 & $1.31 \times 10^{-11}$ \\
\hline >tdbD00011398|Bradyrhizobium_japonicum_USDA_110|224911|Trp|CCA & -28.8 & -27.5 & $5.70 \times 10^{-6}$ \\
\hline >tdbD00005270|Sinorhizobium_meliloti_1021|266834|Gly|GCC & -31.1 & -27.2 & $5.70 \times 10^{-11}$ \\
\hline >tdbD00000036|Acholeplasma_laidlawiil2148|AlalTGC & -29.4 & -26.8 & $1.50 \times 10^{-9}$ \\
\hline >tdbD00008111|Deinococcus_radiodurans_R1|243230|Pro|GGG & -29.5 & -26.6 & $1.16 \times 10^{-4}$ \\
\hline$>$ tdbD00011137|Streptomyces_avermitilis_MA-4680|227882|Val|GAC & -35.5 & -26.5 & $1.16 \times 10^{-4}$ \\
\hline >tdbD00010464|Pyrococcus_horikoshii_OT3|70601|Thr|GGT & -39.4 & -26.4 & $4.11 \times 10^{-4}$ \\
\hline$>$ tdbD00005509|Bartonella_henselae_str._Houston-1|283166|Gly|GCC & -29.6 & -26.4 & $4.85 \times 10^{-2}$ \\
\hline >tdbD00005601|Bartonella_quintana_str._Toulouse|283165|Gly|GCC & -29.6 & -26.4 & $1.06 \times 10^{-3}$ \\
\hline >tdbD00005243|Brucella_melitensis_16M|224914|Gly|GCC & -29.5 & -26.3 & $5.59 \times 10^{-3}$ \\
\hline$>$ tdbD00005264|Agrobacterium_tumefaciens_str._C58|176299|Gly|GCC & -29.5 & -26.3 & $1.20 \times 10^{-5}$ \\
\hline >tdbD00005526|Rhodopseudomonas_palustris_CGA009|258594|Gly|GCC & -29.5 & -26.3 & $1.21 \times 10^{-3}$ \\
\hline >tdbD00000843|Azorhizobium_caulinodans|7|Gly|GCC & -29.5 & -26.3 & $3.94 \times 10^{-5}$ \\
\hline >tdbD00003876|Caenorhabditis_elegans|6239|AlalAGC & -28.4 & -26.3 & $1.80 \times 10^{-5}$ \\
\hline >tdbD00005263|Agrobacterium_tumefaciens_str._C58|176299|Gly|GCC & -29.5 & -26.3 & $5.74 \times 10^{-3}$ \\
\hline >tdbD00008529|Aquifex_aeolicus_VF5|224324|GIn|TTG & -32.8 & -26.3 & $5.74 \times 10^{-3}$ \\
\hline >tdbD00005411|Brucella_suis_1330|204722|Gly|GCC & -29.5 & -26.3 & $6.49 \times 10^{-8}$ \\
\hline >tdbD00000037|Acholeplasma_laidlawiil2148|AlalTGC & -31.8 & -26.1 & $3.88 \times 10^{-7}$ \\
\hline >tdbD00005456|Tropheryma_whipplei_str._Twist|203267|Gly|GCC & -29.4 & -26 & $3.88 \times 10^{-7}$ \\
\hline >tdbD00005459|Tropheryma_whipplei_TW08/27|218496|Gly|GCC & -29.4 & -26 & $1.46 \times 10^{-4}$ \\
\hline >tdbD00010458|Pyrococcus_abyssi_GE5|272844|Thr|GGT & -39.9 & -26 & $1.68 \times 10^{-4}$ \\
\hline >tdbD00005209|Thermotoga_maritima_MSB8|243274|GlylCCC & -33.7 & -26 & $1.34 \times 10^{-5}$ \\
\hline >tdbD00010375|Thermotoga_maritima_MSB8|243274|Thr|CGT & -33.1 & -26 & $2.02 \times 10^{-5}$ \\
\hline >tdbD00010461|Pyrococcus_furiosus_DSM_3638|186497|Thr|GGT & -39.9 & -26 & $6.98 \times 10^{-5}$ \\
\hline >tdbD00012004|Nanoarchaeum_equitans_Kin4-M|228908|Lys|TTT & -38.1 & -25.9 & $2.53 \times 10^{-4}$ \\
\hline >tdbD00003695|Buchnera_aphidicola_str._APS_(Acyrthosiphon_pisum)|107806|Ala|TGC & -29.3 & -25.8 & $7.06 \times 10^{-4}$ \\
\hline >tdbD00003931|Yersinia_pestis_KIM|187410|AlalTGC & -29.3 & -25.8 & $2.85 \times 10^{-4}$ \\
\hline >tdbD00003953|Shewanella_oneidensis_MR-1|211586|AlalTGC & -29.1 & -25.8 & $4.77 \times 10^{-5}$ \\
\hline >tdbD00004024|Photorhabdus_luminescens_subsp._laumondii_TTO1|243265|Ala|TGC & -29.3 & -25.8 & $2.26 \times 10^{-4}$ \\
\hline >tdbD00004054|Yersinia_pseudotuberculosis_IP_32953|273123|AlalTGC & -29.3 & -25.8 & $4.04 \times 10^{-4}$ \\
\hline >tdbD00003903|Buchnera_aphidicola_str._Sg_(Schizaphis_graminum)|198804|Ala|TGC & -29.3 & -25.8 & $1.84 \times 10^{-5}$ \\
\hline >tdbD00004052|Yersinia_pestis_biovar_Microtus_str._91001|229193|Ala|TGC & -29.3 & -25.8 & $1.71 \times 10^{-4}$ \\
\hline
\end{tabular}


Table 9 continued

\begin{tabular}{|c|c|c|c|}
\hline geneID & $\begin{array}{l}\mathrm{CL} \Delta G \\
(\mathrm{kcal} / \mathrm{mol})\end{array}$ & $\begin{array}{l}\mathrm{DH} \Delta G \\
(\mathrm{kcal} / \mathrm{mol})\end{array}$ & $\begin{array}{l}\text { Expected } \mathrm{CI} / \mathrm{CL} \\
\text { frequency ratio }\end{array}$ \\
\hline$>$ tdbD00003775|Yersinia_pestis_CO92/214092|Ala|TGC & -29.3 & -25.8 & $3.17 \times 10^{-6}$ \\
\hline >tdbD00008312|Leptospira_interrogans_serovar_Lai_str._56601|189518|Pro|GGG & -27 & -25.8 & $3.30 \times 10^{-10}$ \\
\hline >tdbD00010466|Sulfolobus_solfataricus_P2|273057|Thr|CGT & -39.5 & -25.7 & $1.81 \times 10^{-5}$ \\
\hline
\end{tabular}

Table 10 Top 50 stable CI structures in actual tRNA sequences

\begin{tabular}{|c|c|c|c|}
\hline geneID & $\begin{array}{l}\text { CL } \Delta \mathrm{G} \\
(\mathrm{kcal} / \mathrm{mol})\end{array}$ & $\begin{array}{l}\text { CI } \Delta \mathrm{G} \\
(\mathrm{kcal} / \mathrm{mol})\end{array}$ & $\begin{array}{l}\text { Expected } \mathrm{CI} / \mathrm{CL} \\
\text { frequency ratio }\end{array}$ \\
\hline >tdbD00012048|Staphylothermus_marinus_F1|399550|Arg|GCG & -37.8 & -28.24 & $1.83 \times 10^{-7}$ \\
\hline >tdbD00012086|Sulfolobus_acidocaldarius_DSM_639|330779|Val|CAC & -32 & -27.89 & $1.27 \times 10^{-3}$ \\
\hline >tdbD00012046|Staphylothermus_marinus_F1|399550|Arg|CCT & -37.8 & -27.74 & $8.15 \times 10^{-8}$ \\
\hline >tdbD00011538|Pyrobaculum_aerophilum_str._IM2|178306|Ini|CAT & -38.7 & -27.67 & $1.69 \times 10^{-8}$ \\
\hline >tdbD00012016|Staphylothermus_marinus_F1|399550|Arg|TCG & -37.7 & -25.64 & $3.18 \times 10^{-9}$ \\
\hline$>$ tdbD00012034|Staphylothermus_marinus_F1|399550|Arg|TCT & -37.6 & -25.44 & $2.70 \times 10^{-9}$ \\
\hline >tdbD00011053|Sulfolobus_tokodaii_str._7|273063IVallTAC & -34.3 & -25 & $2.80 \times 10^{-7}$ \\
\hline >tdbD00011052|Sulfolobus_tokodaii_str._7|273063|VallGAC & -34.2 & -24.9 & $2.80 \times 10^{-7}$ \\
\hline >tdbD00004666|Sinorhizobium_meliloti_1021|266834|GlulTTC & -30.2 & -24.34 & $7.43 \times 10^{-5}$ \\
\hline$>$ tdbD00011994|Nanoarchaeum_equitans_Kin4-M|228908|Pro|TGG & -36.1 & -24.04 & $3.18 \times 10^{-9}$ \\
\hline$>$ tdbD00002413|Archaeoglobus_fulgidus|2234|Arg|TCG & -33.3 & -23.8 & $2.02 \times 10^{-7}$ \\
\hline$>$ tdbD00009035|Archaeoglobus_fulgidus_DSM_4304|224325|Arg|TCG & -33.3 & -23.8 & $2.02 \times 10^{-7}$ \\
\hline$>$ tdbD00007425|Methanothermobacter_thermautotrophicus_str._Delta_HI187420|Met|CAT & -29.2 & -23.6 & $1.13 \times 10^{-4}$ \\
\hline$>$ tdbD00003102|Sulfolobus_solfataricus|2287|Val|GAC & -32.7 & -23.4 & $2.80 \times 10^{-7}$ \\
\hline$>$ tdbD00012089|Sulfolobus_acidocaldarius_DSM_639|330779|Val|GAC & -32.7 & -23.4 & $2.80 \times 10^{-7}$ \\
\hline$>$ tdbD00005705|Methanothermobacter_thermautotrophicus_str._Delta_HI187420|His|GTG & -24.4 & -23.3 & 0.168 \\
\hline >tdbD00012032|Staphylothermus_marinus_F1|399550|Gly|TCC & -34.9 & -23.2 & $5.70 \times 10^{-9}$ \\
\hline >tdbD00004311|Bartonella_henselae_str._Houston-1|283166|Cys|GCA & -22.7 & -23.1 & 1.91 \\
\hline >tdbD00009062|Pyrococcus_furiosus_DSM_3638|186497|ArgITCG & -36 & -23.04 & $7.37 \times 10^{-10}$ \\
\hline >tdbD00003824|Thermoplasma_acidophilum_DSM_1728|273075|Ala|CGC & -31.4 & -22.9 & $1.02 \times 10^{-6}$ \\
\hline >tdbD00003827|Thermoplasma_volcanium_GSS1|273116|AlalCGC & -31.4 & -22.9 & $1.02 \times 10^{-6}$ \\
\hline >tdbD00012041|Staphylothermus_marinus_F1|399550|Thr|GGT & -36.5 & -22.74 & $2.01 \times 10^{-10}$ \\
\hline >tdbD00012006INanoarchaeum_equitans_Kin4-M|228908|AlalTGC & -35.8 & -22.7 & $5.87 \times 10^{-10}$ \\
\hline >tdbD00012010|Nanoarchaeum_equitans_Kin4-M|228908|Ala|CGC & -35.8 & -22.7 & $5.87 \times 10^{-10}$ \\
\hline >tdbD00011999|Nanoarchaeum_equitans_Kin4-M|228908|Ala|GGC & -35.7 & -22.6 & $5.87 \times 10^{-10}$ \\
\hline >tdbD00012090|Sulfolobus_acidocaldarius_DSM_639|330779|Val|TAC & -31.7 & -22.5 & $3.29 \times 10^{7}$ \\
\hline >tdbD00005896|Agrobacterium_tumefaciens_str._C58|176299|Ile|GAT & -33.2 & -22.4 & $2.45 \times 10^{-8}$ \\
\hline$>$ tdbD00005281|Methanothermobacter_thermautotrophicus_str._Delta_H|187420|Gly|TCC & -29.8 & -22.39 & $6.00 \times 10^{-6}$ \\
\hline >tdbD00009055|Pyrococcus_abyssi_GE5|272844|Arg|CCT & -36.4 & -22.34 & $1.24 \times 10^{-10}$ \\
\hline >tdbD00003900|Methanosarcina_mazei_Go1|192952|AlalGGC & -29.7 & -22.27 & $5.81 \times 10^{-6}$ \\
\hline >tdbD00011889|Methanosarcina_acetivorans_C2Al188937|AlalGGC & -29.7 & -22.27 & $5.81 \times 10^{-6}$ \\
\hline >tdbD00009027|Aeropyrum_pernix_K1|272557|Arg|CCG & -37.9 & -22.14 & $7.85 \times 10^{-12}$ \\
\hline >tdbD00008210|Thermoplasma_volcanium_GSS1|273116|ProlTGG & -30.9 & -22.09 & $6.19 \times 10^{-7}$ \\
\hline >tdbD00008207|Thermoplasma_acidophilum_DSM_1728|273075|ProlTGG & -30.9 & -22.09 & $6.19 \times 10^{-7}$ \\
\hline$>$ tdbD00011976|Nanoarchaeum_equitans_Kin4-M|228908|Gly|GCC & -35.5 & -21.9 & $2.61 \times 10^{-10}$ \\
\hline$>$ tdbD00009045|Methanothermobacter_thermautotrophicus_str._Delta_HI187420|Arg|TCT & -31.5 & -21.84 & $1.56 \times 10^{-7}$ \\
\hline >tdbD00012005|Nanoarchaeum_equitans_Kin4-M|228908|Pro|GGG & -39.8 & -21.64 & $1.60 \times 10^{-13}$ \\
\hline >tdbD00010969|Pyrococcus_abyssi_GE5|272844|Val|TAC & -34.8 & -21.6 & $4.99 \times 10^{-10}$ \\
\hline >tdbD00010972|Pyrococcus_furiosus_DSM_3638|186497|VallTAC & -34.8 & -21.6 & $4.99 \times 10^{-10}$ \\
\hline
\end{tabular}


Table 10 continued

\begin{tabular}{|c|c|c|c|}
\hline geneID & $\begin{array}{l}\mathrm{CL} \Delta \mathrm{G} \\
(\mathrm{kcal} / \mathrm{mol})\end{array}$ & $\begin{array}{l}\text { CI } \Delta \mathrm{G} \\
(\mathrm{kcal} / \mathrm{mol})\end{array}$ & $\begin{array}{l}\text { Expected CI/CL } \\
\text { frequency ratio }\end{array}$ \\
\hline >tdbD00011989|Nanoarchaeum_equitans_Kin4-M|228908IVal|GAC & -35.2 & -21.5 & $2.22 \times 10^{-10}$ \\
\hline >tdbD00012003INanoarchaeum_equitans_Kin4-M|228908IVallTAC & -35.2 & -21.5 & $2.22 \times 10^{-10}$ \\
\hline >tdbD00011992INanoarchaeum_equitans_Kin4-M|228908IVal|CAC & -35.2 & -21.5 & $2.22 \times 10^{-10}$ \\
\hline >tdbD00009060|Pyrococcus_furiosus_DSM_3638|186497|Arg|CCT & -36.4 & -21.34 & $2.44 \times 10^{-11}$ \\
\hline >tdbD00007448|Thermoplasma_acidophilum_DSM_1728|273075|Met|CAT & -32.2 & -21.2 & $1.77 \times 10^{-8}$ \\
\hline >tdbD00004505|Streptococcus_mutans_UA159|210007|Asp|GTC & -28.9 & -21.1 & $3.19 \times 10^{-6}$ \\
\hline >tdbD00002929|Thermococcus_celer|2264|Thr|GGT & -38.2 & -21.04 & $8.09 \times 10^{-13}$ \\
\hline >tdbD00004898|Takifugu_rubripes|31033|Glu|TTC & -24.2 & -20.9 & $4.73 \times 10^{-3}$ \\
\hline >tdbD00005387|Sulfolobus_tokodaii_str._7|273063|Gly|GCC & -32 & -20.84 & $1.37 \times 10^{-8}$ \\
\hline >tdbD00008197|Pyrococcus_furiosus_DSM_3638|186497|ProlGGG & -33.4 & -20.7 & $1.12 \times 10^{-9}$ \\
\hline >tdbD00004892|Takifugu_rubripes|31033|Glu|CTC & -28.1 & -20.7 & $6.10 \times 10^{-6}$ \\
\hline
\end{tabular}

